\title{
Linked color imaging improves the endoscopic visibility of gastric mucosal cancers
}

\section{다 (1) $\odot$}

\author{
Authors \\ Imazeki $^{3}$, Taketo Yamaguchi ${ }^{3}$ \\ Institutions \\ 1 Endoscopy Division, Chiba Cancer Center, Chiba, Japan \\ 2 Hara Clinic, Chiba, Japan \\ 3 Department of Gastroenterology, Chiba Cancer Center, \\ Chiba, Japan
}

Yoshiyasu Kitagawa ${ }^{1}$, Takuto Suzuki ${ }^{1}$, Taro Hara ${ }^{2}$, Rino Nankinzan ${ }^{1}$, Hideyuki Takashiro ${ }^{1}$, Osamu Sugita ${ }^{1}$, Hiroshi

submitted 25.1.2018

accepted after revision 11.6 .2018

\section{Bibliography}

DOI https://doi.org/10.1055/a-0733-7086

Endoscopy International Open 2019; 07: E164-E170

(c) Georg Thieme Verlag KG Stuttgart · New York

ISSN 2364-3722

Corresponding author

Yoshiyasu Kitagawa, MD, Endoscopy Division, Chiba Cancer Center, 666-2 Nitonacho, Chuo-ku, Chiba, Japan

Fax: +043-262-8680

ykitagawa@chiba-cc.jp

\section{ABSTRACT}

Background and study aims As a newly developed endoscopy technique, linked color imaging $(\mathrm{LCl})$ provides very bright images with enhanced color tones. With the ob- jective of improving the detection rate of gastric mucosal cancers, which are often difficult to detect, we examined the utility of $\mathrm{LCl}$ from the viewpoint of visibility.

Patients and methods The current study used 100 consecutive gastric mucosal cancers $\leq 20 \mathrm{~mm}$ in diameter. For each lesion, we selected one endoscopic image acquired by white-light imaging (WLI), blue-laser imaging (BLI) -bright, and $\mathrm{LCl}$ modes. Four endoscopists interpreted the images; using a previously reported scale, we scored the visibility level on a scale of $1-4$.

Results The mean $( \pm S D$ ) visibility scores were $2.54 \pm 1.10$ for WLI, $3.02 \pm 1.07$ for BLI-bright, and $3.28 \pm 0.97$ for LCI. The score was significantly higher for BLI-bright compared with WLI $(P<.001)$ and again higher for $\mathrm{LCl}$ compared with BLI-bright $(P<.001)$. For the experts, the scores for BLIbright and $\mathrm{LCl}$ were similar, but both were significantly higher than the score for WLI. For the trainees, there was no significant difference between the WLI and BLI-bright scores, but $\mathrm{LCl}$ score was significantly higher than those for WLI and BLI-bright scores. With regard to clinical characteristics, LCl particularly enhanced visibility of normochromic, flat and depressed lesions, which had the lowest visibility scores of all three modalities compared with those of the other lesions.

Conclusion $\mathrm{LCl}$ increased visibility and may contribute to early detection of gastric mucosal cancers.

\section{Introduction}

Gastric cancer is the second most common cause of cancerassociated deaths worldwide [1]; therefore, early detection and curative treatment are the best strategies to improve patient survival. Detection of gastric cancers $\leq 20 \mathrm{~mm}$ in diameter is particularly ideal because they are curable by minimally invasive treatment modalities, including endoscopic mucosal resection and endoscopic submucosal dissection [2,3]. Esophagogastroduodenoscopy (EGD) is the most sensitive method for early detection of gastric cancers, but it is often difficult while using conventional white-light imaging (C-WLI) endoscopy [4]. Hence, new modalities are required.
In recent years, image-enhanced endoscopy (IEE), including narrow-band imaging (NBI) and blue-laser imaging (BLI), has been used to improve visualization of microstructures and microvessels in gastrointestinal lesions and to increase diagnostic accuracy [5-7]. Previous studies have shown that conventional IEE, including NBI and BLI, was effective for early detection of neoplastic lesions in the esophagus and colon [8-10]. Although conventional IEE was also expected to facilitate early detection of gastric mucosal cancers, no studies have demonstrated its utility in this regard; this lack of evidence may be because conventional IEE probably does not provide sufficient brightness for effective examination of the stomach, which has a wider lumen compared with the esophagus and colon. 


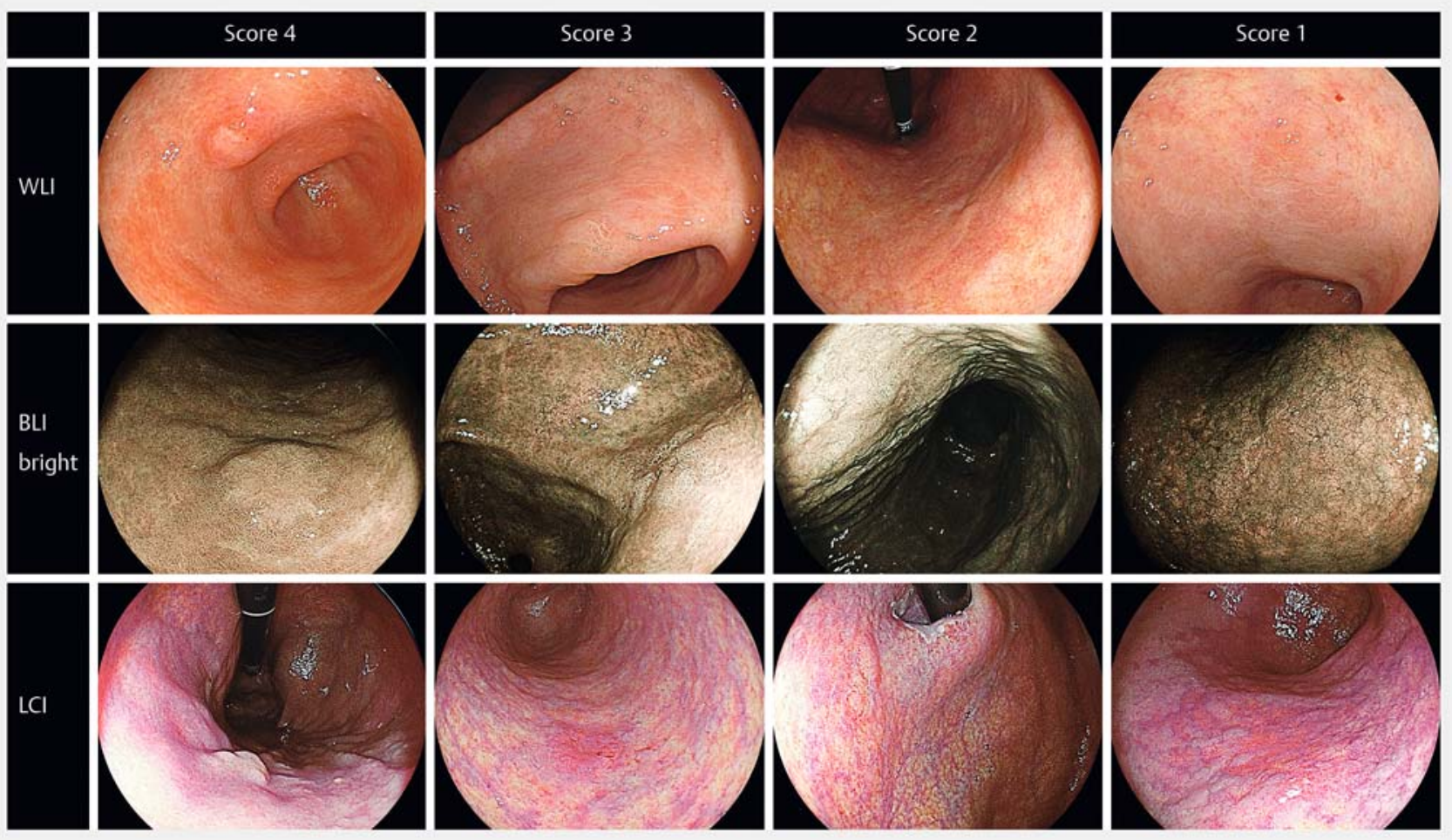

Fig. 1 Representative lesions with visibility scores 1 - 4 using the three modalities. WLI, white-light imaging; BLI-bright, blue-laser imagingbright; LCI, linked color imaging.

Linked color imaging (LCI) (Fujifilm Co., Tokyo, Japan) is a newly developed IEE modality that provides images with enhanced brightness and visualization of red lesions and whitish lesions during routine endoscopy. Another merit is that $\mathrm{LCl}$ images resemble color-enhanced C-WLI images; thus, eliminating the need for special training. We reported that $\mathrm{LCl}$ increases visibility of flat colorectal lesions [11]. Previous studies, which were case series involving a small number of patients, reported that LCl enhances early gastric cancer lesions [12,13]. However, the efficacy of this modality compared with conventional IEE remains unknown.

With the aim of examining the utility of $\mathrm{LCl}$ in improving detection of gastric mucosal cancer, we conducted a retrospective study to compare three modalities, including WLI, BLI-bright, and $\mathrm{LCl}$, with regard to their ability to facilitate the visualization of gastric mucosal cancer.

\section{Patients and methods}

Between December 2014 and July 2017, 113 consecutive early gastric cancers were examined by EGD and were histologically proven mucosal cancers that measured $\leq 20 \mathrm{~mm}$ in diameter at Chiba Cancer Center, Chiba, Japan. One expert (Y.K.) took all the endoscopic images. Thirteen cases examined using a NBI system (Olympus Medical Systems, Tokyo, Japan) were excluded from the analysis. Thus, the current study used endoscopic images of 100 early gastric cancers, examined using a LASEREO system (FUJIFILM, Tokyo, Japan) with an EG-L590ZW or L600ZW endoscope. For all lesions, we selected one image each acquired by WLI, BLI-bright, and LCI from the same site or from the intermediate or distant view. A total of 100 images were prepared for each modality.

Before the study, surface colors in WLI were evaluated by two experts (Y. K. and T.H.) and then divided into three groups; whitish, normochromic, and reddish lesions. They were diagnosed based on the predominant color. The endoscopic images of WLI, BLI-bright, and LCI were randomly displayed and independently reviewed by four endoscopists, including two experts and two trainees, in a single session without prior knowledge of the lesions. In this study, the endoscopists were defined as experts when their experience in using IEE was over 5 years or as trainees when their experience was less than 1 year. Images were electronically presented without zooming; image size was predetermined and similar to that used during routine endoscopic monitoring. The reviewers did not refer to earlier images. They scored the lesions based on a previously reported 4point visibility scale $[11,14]$, as follows: score 4 , excellent (easily detectable); score 3 , good (detectable with careful observation); score 2, fair (hardly detectable without careful examination); and score 1, poor (not detectable without repeated careful examination). Previous study demonstrated that this score was related to an objective indicator such as color difference value [15]. A representative image for each score is shown in - Fig. 1 .

The current study was approved by the ethics committee of Chiba Cancer Center and the contents were displayed on the 
notice board for inpatients and outpatients. The study was carried out in accordance with the World Medical Association's Declaration of Helsinki, and all the patients provided informed consent for undergoing EGD.

\section{Histopathologic diagnosis}

Lesion specimens were obtained by endoscopic resection and were fixed with $10 \%$ formalin. Histopathological diagnosis was performed according to the Japanese Classification of Gastric Carcinoma [16].

\section{$\mathrm{LCl}$, the novel image-enhancing mode}

We used an endoscopic system employing a laser light source (LASEREO), which has two lasers with different wavelengths; white-light laser (wavelength $450 \pm 10 \mathrm{~nm}$ ) provides a wide spectrum of white light illumination, suitable for general observation, whereas BLI mode laser comprises short wavelength $(410 \pm 10 \mathrm{~nm})$ and narrow band. BLI mode provides high contrast signals to obtain information regarding capillaries on the mucosal surface, slight mucosal irregularities, and deep blood vessels. The intermediate mode BLI-bright, which has a higher white light intensity ratio, provides a brighter image. $\mathrm{LCl}$ used in the current study was a novel image-enhanced mode based on BLI-bright imaging with additional processing that enhanced red color separation to depict red and white colors more vividly. Because $\mathrm{LCl}$ enhanced color contrast, color difference between the surrounding tissue and lesions, including light-colored lesions, is emphasized, thereby facilitating visualization.

\section{Statistical analysis}

Clinical data were expressed as percentage, mean, and range. The mean $( \pm S D)$ of the visibility scores was calculated. Mean scores of all endoscopists, the expert group, and the trainee group were analyzed and compared among the modalities using paired $t$-test. The modalities were also compared in terms of various clinical characteristics, including surface color and macroscopic type. $P$ values $<0.05$ were considered to be significant. Statistical analyses were performed using SPSS software, version 17.0 (SPSS Inc, Chicago, IL).

\section{Results}

Patient characteristics are summarized in $>$ Table 1. Median patient age was $74.5(62-90)$ years, with a preponderance of men $(\mathrm{n}=77)$. Median lesion size was $10 \mathrm{~mm}(2-20 \mathrm{~mm})$, and surface colors were whitish in 16, normochromic in 55, and reddish in 29 lesions. Macroscopic types were as follows: protruding in 33 , flat in 14, and depressed in 53. Gastric lesions were located in the upper third in 17 patients, in the middle third in $16 \mathrm{pa}$ tients, and in the lower third in 67 patients. There were 94 cases of well-differentiated tubular adenocarcinoma and six cases of moderately differentiated tubular adenocarcinoma. Representative cases are shown in $>$ Fig. 2, > Fig.3, > Fig.4, and $>$ Fig. $\mathbf{5}$.

Mean $( \pm S D)$ visibility scores of the four endoscopists were $2.54 \pm 1.10$ for WLI, $3.02 \pm 1.07$ for BLI-bright, and 3.28 \pm 0.97 for $\mathrm{LCl}$. The score for BLI-bright was significantly higher than
- Table 1 Baseline clinicopathologic characteristics.

\begin{tabular}{|l|c|}
\hline No. of patients & 100 \\
\hline Male/female, no. & $77 / 23$ \\
\hline Median age (range), years & $74.5(62-90)$ \\
\hline Lesion size, median (range), mm & $10.0(2-20)$ \\
\hline Surface color & \\
\hline - Whitish & 16 \\
\hline - Normochromic & 55 \\
\hline - Reddish & 29 \\
\hline Macroscopic type & 33 \\
\hline - Elevated & 14 \\
\hline - Flat & 53 \\
\hline - Depressed & \\
\hline Location of the gastric lesion & 17 \\
\hline - Upper third & 16 \\
\hline - Middle third & 67 \\
\hline - Lower third & 64 \\
\hline Histology & \\
\hline - Tub1 & \\
\hline - Tub2 & \\
\hline - & \\
\hline
\end{tabular}

that for WLI $(P<0.001)$ and the score for $\mathrm{LCl}(P<0.001)$ was significantly higher than that for BLI-bright mode. For the experts, the score for BLI-bright was significantly higher than that for WLI $(3.23 \pm 0.95$ vs. $2.51 \pm 1.08$; $P<0.001)$, but there was no difference between the scores for BLI-bright and $\mathrm{LCl}(3.23 \pm 0.95$ vs. $3.38 \pm 0.83)$. For the trainees, there was no difference between the scores for WLI $(2.57 \pm 1.12)$ and BLI-bright $(2.79 \pm$ $1.13)$, but the score for $\mathrm{LCl}(3.18 \pm 1.08)$ was significantly higher than that for WLI and BLI-bright $(P<0.001)$ ( $\triangleright$ Table 2$)$.

Distribution of scores for the three modalities is shown in - Fig. 6. The proportion of cases with scores 3 and 4, which represented good visibility, was $50.3 \%$ for WLI compared with $79 \%$ for LCl. Conversely, the proportion of cases with scores 1 and 2, which represented poor visibility, was $49.8 \%$ for WLI compared with $21 \%$ for LCl. Next, when the mean score of the four endoscopists was calculated for each lesion, 28 cases had mean scores of 2 or lower on WLI, 15 (53.6\%) of which had scores of 3 or higher on $\mathrm{LCl}$. Among 13 cases with scores of 2 or lower on BLI-bright, 3 cases (23.1\%) showed scores of 3 or higher on LCl. Based on the assessment of four endoscopists, two lesions had mean scores of 2 or lower on $\mathrm{LCl}$, but neither of the two showed scores of 3 or higher on WLI or BLI-bright.

Mean visibility scores of the four endoscopists, in terms of various clinical characteristics including surface color and macroscopic type, are shown in $>$ Table 3 . With regard to surface 

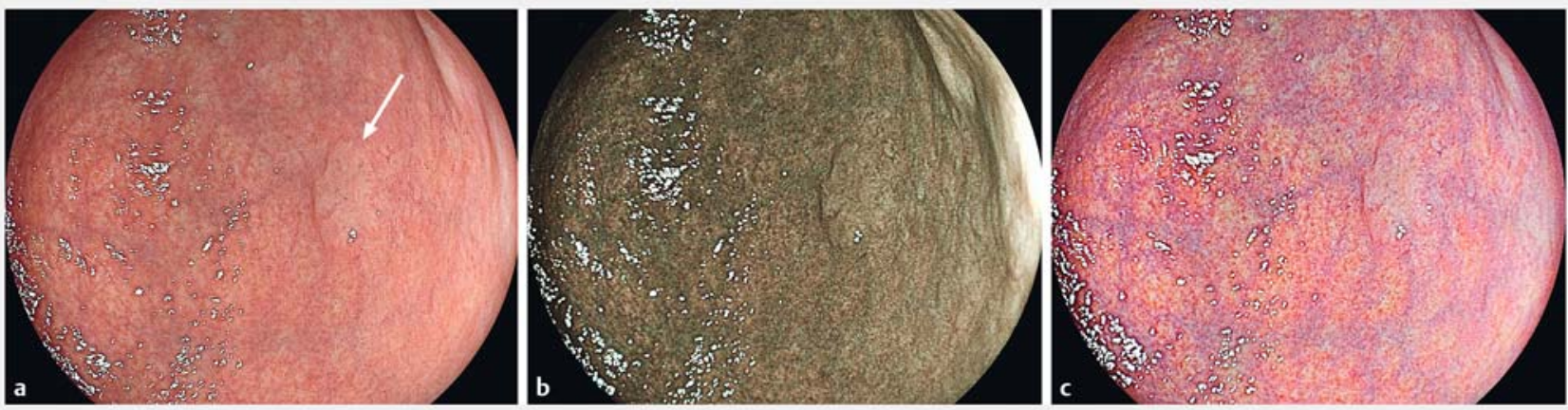

Fig. 2 a White-light imaging shows a whitish lesion (arrow) in the lower third of the stomach: mean visibility score, 3.75. b Blue-laser imaging-bright: mean visibility score, 3.50. c Linked color imaging enables enhanced visualization of the whitish lesion: mean visibility score, 4.00 .
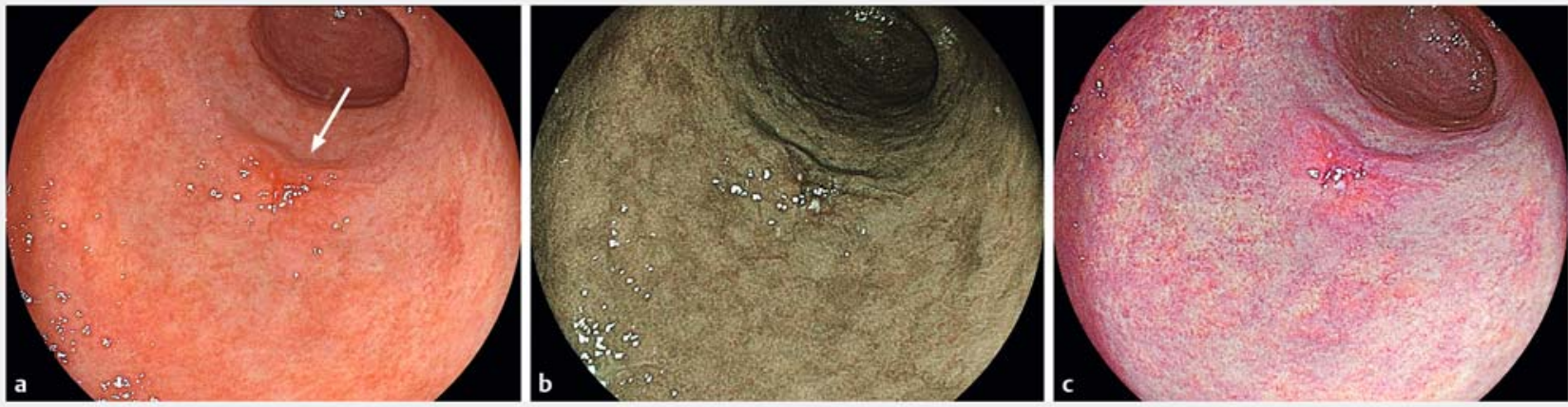

- Fig. 3 a White-light imaging shows a reddish lesion (arrow) in the lower third of the stomach: mean visibility score, 3.50. b Blue-laser imaging-bright: mean visibility score, 3.75. c Linked color imaging enables enhanced visualization of the reddish lesion: mean visibility score, 4.00.
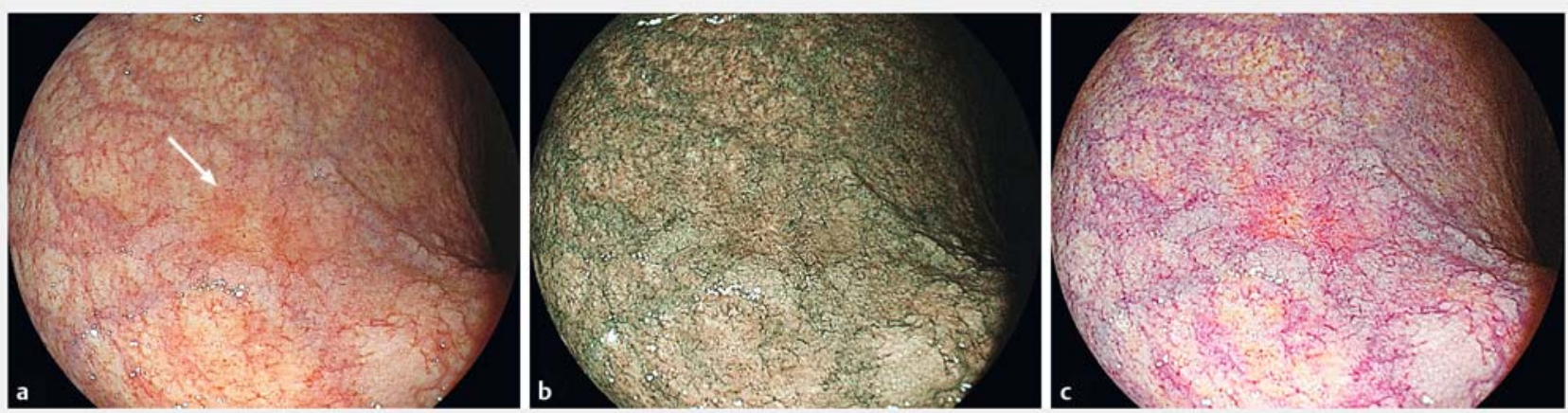

- Fig. 4 Endoscopic images of the same gastric mucosal cancer (case \#1). a White-light imaging shows a depressed lesion (arrow) in the upper third of the stomach: mean visibility score, 1.75. b Blue-laser imaging-bright: mean visibility score, 2.75. c Linked color imaging, which provides a high color contrast image to vividly differentiate between the cancerous lesion and the surrounding normal mucosa: mean visibility score, 3.50 .

color, mean ( \pm SD) visibility scores for WLI, BLI-bright, and LCI in normochromic lesions were $2.15 \pm 1.03,2.78 \pm 1.10$, and $3.08 \pm$ 1.04 , respectively, which were lower those for whitish lesions $(3.14 \pm 1.10,3.47 \pm 0.93$, and $3.69 \pm 0.64$, respectively) and reddish lesions $(2.95 \pm 0.91,3.23 \pm 0.97$, and $3.42 \pm 0.88$, respec- tively). These findings indicated that in normochromic lesions, although visibility scores for all three modalities were lower than those in whitish and reddish lesions, the score for $\mathrm{LCl}$ remained significantly higher than those for the other two modalities. With regard to macroscopic type, mean $( \pm S D)$ visibility 

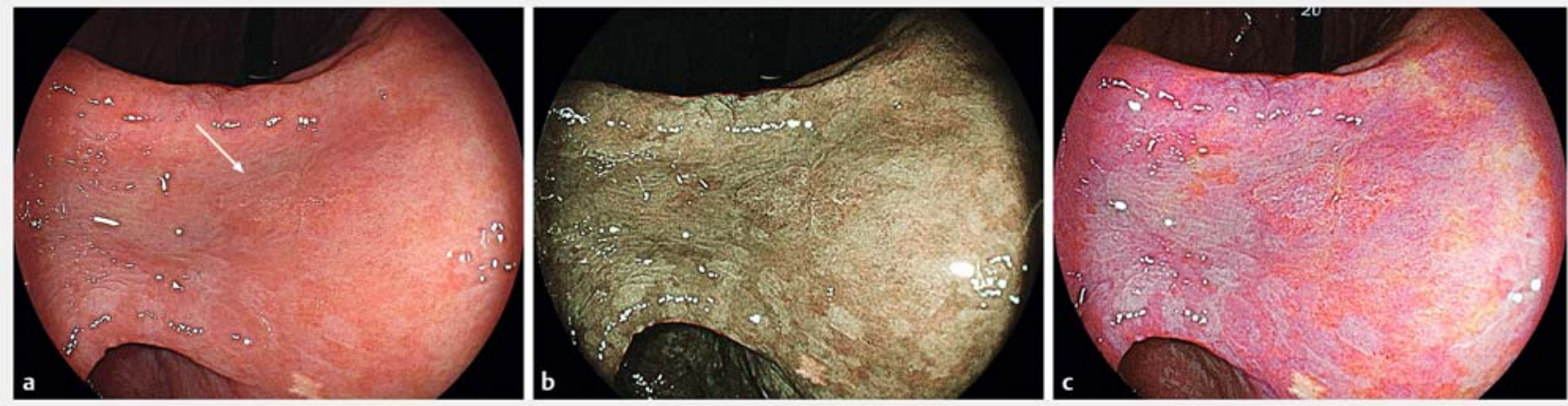

Fig. 5 Endoscopic images of the same gastric mucosal cancer (case \#2). a White-light imaging shows a flat lesion (arrow) in the lower third of the stomach: mean visibility score, 2.25. b Blue-laser imaging-bright: mean visibility score, 2.75. c Linked color imaging, which enhances mucosal irregularity: mean visibility score, 3.00.

- Table 2 Mean visibility scores of endoscopists (experts and trainees) for WLI, BLI-bright, and LCI modalities.

\begin{tabular}{|l|l|l|l|l|l|l|}
\hline Endoscopist & WLI & BLI-bright & LCI & $\begin{array}{l}\text { WLI vs. BLI-bright, } \\
\text { P value }\end{array}$ & $\begin{array}{l}\text { WLI vs. LCI, } \\
\boldsymbol{P} \text { value }\end{array}$ & $\begin{array}{l}\text { BLI-bright vs. LCI, } \\
\boldsymbol{P} \text { value }\end{array}$ \\
\hline All, mean \pm SD & $2.54 \pm 1.10$ & $3.02 \pm 1.07$ & $3.28 \pm 0.97$ & $<0.001$ & $<0.001$ & $<0.001$ \\
\hline Expert, mean \pm SD & $2.51 \pm 1.08$ & $3.23 \pm 0.95$ & $3.38 \pm 0.83$ & $<0.001$ & $<0.001$ & $\mathrm{NS}$ \\
\hline Trainee, mean \pm SD & $2.57 \pm 1.12$ & $2.79 \pm 1.13$ & $3.18 \pm 1.08$ & NS & $<0.001$ & $<0.001$ \\
\hline WLI, white-light imaging; BLI, blue-laser imaging; LCI, linked color imaging; SD, standard deviation; NS, not significant.
\end{tabular}

\begin{tabular}{|c|c|c|c|c|c|c|c|c|c|}
\hline \multirow[b]{2}{*}{ BLI-bright } & \multicolumn{3}{|c|}{88} & \multicolumn{2}{|l|}{111} & \multicolumn{2}{|l|}{98} & \multicolumn{2}{|l|}{103} \\
\hline & 57 & & 53 & 11 & & & 17 & & \\
\hline \multirow[t]{4}{*}{$\mathrm{LCl}$} & 31 & 53 & \multicolumn{3}{|c|}{89} & \multicolumn{4}{|c|}{227} \\
\hline & 0 & 50 & 100 & 150 & 200 & 250 & 300 & 350 & 400 \\
\hline & \multicolumn{9}{|c|}{ Numbers } \\
\hline & Visib & ility & core & 1 & 2 & 3 & 4 & & \\
\hline
\end{tabular}

Fig. 6 Distribution of visibility scores. For each modality, the total scores of all endoscopists were counted. WLI, white-light imaging; BLI-bright, blue-laser imaging-bright; LCl, linked color imaging.

scores in flat and depressed lesions were $2.33 \pm 1.04$ for WLI, $2.84 \pm 1.09$ for BLI-bright, and $3.15 \pm 1.02$ for $\mathrm{LCI}$, which were lower than the scores in protruding lesions $(2.95 \pm 1.11,3.39 \pm$ 0.93 , and $3.53 \pm 0.80$, respectively). These findings showed that in flat and depressed lesions, although visibility scores of all three modalities were lower than those in protruding lesions, the score for $\mathrm{LCl}$ remained significantly higher than those for the other two modalities.

\section{Discussion}

In the current study, we proved that, compared with WLI and BLI-bright modes, LCI mode improved visibility of gastric mucosal cancers measuring $\leq 20 \mathrm{~mm}$ in diameter. Upon comparing visibility scores of experts with those of trainees, those for BLIbright and LCl were significantly higher than those for WLI, with no significant difference between the scores for BLI-bright and $\mathrm{LCl}$ in experts; conversely, in trainees, the score for $\mathrm{LCl}$ was significantly higher than the scores for WLI and BLI-bright, with no significant difference between WLI and BLI-bright. One possible reason for these results was that the images of conventional IEE, such as BLI-bright, had completely different color tones compared with those of standard WLI and that some familiarization was required. In contrast, because $\mathrm{LCI}$ images resembled color-enhanced WLI images, $\mathrm{LCl}$ may have a steep learning curve and is easy to adopt even in facilities with limited opportunities to diagnose early gastric cancer. Therefore, this modality could be extremely useful in actual clinical practice, may enable IEE for observation, and can potentially become the standard method for detection of gastric cancer.

In this study, distribution of surface colors was similar to that reported in a previous study [17]. With regard to surface color, the score for $\mathrm{LCl}$ in normochromic lesions was significantly higher than those for WLI and BLI-bright. This is because LCl enhances color separation and depicts red lesions even redder and white lesions whiter, which contributes to demonstrating large color differences in color contrast between a malignant lesion and the surrounding area, even if poor tone difference is 
- Table 3 Mean visibility scores of endoscopists, in terms of various clinical characteristics.

\begin{tabular}{l|l|l|l|l|l|l|}
\hline & WLI & BLI-bright & LCI & $\begin{array}{l}\text { WLI vs. BLI-bright, } \\
\text { P value }\end{array}$ & $\begin{array}{l}\text { WLI vs. LCI, } \\
\text { P value }\end{array}$ \\
\hline \\
\hline Surface color
\end{tabular}

visualized in the other two modalities. In contrast, scores for $\mathrm{LCl}$ in whitish and reddish lesions were not significantly higher than those for BLI-bright. This result may be explained by the imaging principle of BLI-bright, which involves use of light that is easily absorbed by the hemoglobin in capillaries, thereby clearly delineating blood vessels in the reddish lesions as brownish in color. In addition, when a whitish lesion is present, red blood vessels in the surrounding area are also amplified. Interruption of the underlying mucosal vascular pattern provides an indirect finding to facilitate lesion recognition.

In our study, we found that $\mathrm{LCl}$ was effective in enhancing flat and depressed lesions, which may not be visible on WLI because of their subtle appearance and the limited contrast of the surrounding mucosa $[18,19]$. Previous case studies also reported that $\mathrm{LCl}$ enhanced early gastric cancer lesions in patients with these clinical features $[12,13]$. Although BLI-bright provides brighter endoscopic views, the imaging is still criticized for darkness when used in the stomach [20]. Therefore, BLIbright probably does not contribute to detection of subtle changes in early gastric cancer. In contrast, LCl used in the current study yielded very bright images and enhanced mucosal irregularities, even in the middle to distant views, and enabled identification of abnormal lesions. The previous study using same visibility scores also showed that $\mathrm{LCl}$ improved endoscopic visibility of colorectal flat lesions [11]. Thus, the potential benefit of $\mathrm{LCl}$ in detecting flat and depressed lesions may have clinical implications, especially in screening for early gastric cancers.

Early gastric cancer is often overlooked during ordinary endoscopy because it is more difficult to detect than most other upper gastrointestinal tract cancers [4]. The reported rate of missing superficial gastric cancers is $4.6 \%$ to $25.8 \%$ [21-23]. Failure to detect early gastric cancer during endoscopy may contribute to poor prognosis [21]. Thus, a variety of premedication and screening techniques are available to ensure the quality of EGD $[24,25]$. When the distribution of scores was analyzed in our study, the rate of poor visibility of $\mathrm{LCl}$ mode was lower than that of WLI. With regard to the mean score of the four endoscopists for each lesion, over half of the cases that had poor visibility scores on WLI showed good visibility scores on $\mathrm{LCl}$. We supposed that $\mathrm{LCl}$ was advantageous in improving diagnosis of subtle, early gastric cancer.

Recently, some studies concerning efficacy of $\mathrm{LCl}$ for early gastric cancer have been reported $[26,27]$. One report showed objective data on color change, but this study can demonstrate only improved visibility by color deference of each modality [24]. Another report used scores for improvements, which cannot evaluate visibility of baseline modality [25]. Thus, additional reports are required to build consensus about usefulness of $\mathrm{LCl}$, which is a newly developed IEE modality. The strong point of this study is evaluation of each modality including WLI images, which are the basis of comparison. In addition, this study used the visibility scale, which has been used in several reports of IEE. Although this report used the subjective indicator, the result clearly showed a sufficient improvement of LCI for early gastric cancer.

The current study had some limitations. In this study, still images and not videos were reviewed, and the possibility of selection bias persists. The method of consecutive reviews of the same image in different modalities during a single session could have led to another bias. Because this study included only gastric mucosal cancers, further studies are required to clarify whether $\mathrm{LCl}$ is superior to WLI in differentiating between malignant and nonmalignant lesions. Furthermore, this was a single-center retrospective study; a multicenter prospective study may be more accurate in assessing the effectiveness of this new modality for early detection of gastric cancers. Nonetheless, the current study did provide evidence based on a relatively large number of cases that $\mathrm{LCl}$ significantly improved visibility compared with the other modalities under the same conditions.

\section{Conclusion}

In conclusion, $\mathrm{LCl}$ enhanced the visibility of early gastric cancer and may contribute to the early detection of these lesions. Future application of $\mathrm{LCl}$ in gastric screening is anticipated. 
Competing interests

None

References

[1] Ferlay J, Shin HR, Bray F et al. Estimates of worldwide burden of cancer in 2008: GLOBOCAN 2008. Int J Cancer 2010; 15: 2893-2917

[2] Gotoda T, Yanagisawa A, Sasako M et al. Incidence of lymph node metastasis from early gastric cancer: estimation with a large number of cases at two large centers. Gastric Cancer 2000; 3: 219-225

[3] Ono H, Kondo H, Gotoda T et al. Endoscopic mucosal resection for treatment of early gastric cancer. Gut 2001; 48: 225-229

[4] Sekiguchi M, Oda I. High miss rate for gastric superficial cancers at endoscopy: what is necessary for gastric cancer screening and surveillance using endoscopy? Endosc Int Open 2017; 5: E727-E728

[5] Yoshida T, Inoue H, Usui S et al. Narrow-band imaging system with magnifying endoscopy for superficial esophageal lesions. Gastrointest Endosc 2004; 59: 288-295

[6] Ezoe $\mathrm{Y}$, Muto $\mathrm{M}$, Uedo $\mathrm{N}$ et al. Magnifying narrowband imaging is more accurate than conventional white-light imaging in diagnosis of gastric mucosal cancer. Gastroenterology 2011; 141: 2017-2025

[7] Dohi O, Yagi N, Majima A et al. Diagnostic ability of magnifying endoscopy with blue laser imaging for early gastric cancer: a prospective study. Gastric Cancer 2017; 20: 297 - 303

[8] Muto M, Minashi K, Yano T et al. Early detection of superficial squamous cell carcinoma in the head and neck region and esophagus by narrow band imaging: a multicenter randomized controlled trial. J Clin Oncol 2010; 28: 1566 - 1572

[9] Horimatsu T, Sano Y, Tanaka S et al. Next-generation narrow band imaging system for colonic polyp detection: a prospective multicenter randomized trial. Int J Colorectal Dis 2015; 30: 947 - 954

[10] Ikematsu H, Sakamoto T, Togashi K et al. Detectability of colorectal neoplastic lesions using a novel endoscopic system with blue laser imaging: a multicenter randomized controlled trial. Gastrointest Endosc 2017; 86: $386-394$

[11] Suzuki T, Hara T, Kitagawa Y et al. Linked-color imaging improves endoscopic visibility of colorectal nongranular flat lesions. Gastrointest Endosc 2017; 86: 692-697

[12] Fukuda H, Miura Y, Hayashi Y et al. Linked color imaging technology facilitates early detection of flat gastric cancers. Clin J Gastroenterol 2015; 8: 385-389
[13] Ono S, Abiko $S$, Kato M. Linked color imaging enhances gastric cancer in gastric intestinal metaplasia. Dig Endosc 2017; 29: 230-231

[14] Yoshida N, Hisabe T, Hirose R et al. Improvement in the visibility of colorectal polyps by using blue laser imaging. Gastroinetest Endosc 2015; 82: 542-549

[15] Yoshida N, Naito Y, Yasuda R et al. Linked color imaging improves the visibility of various featured colorectal polyps in an endoscopist's visibility and color difference value. Int J Colorectal Dis 2017; 32: 1253 1260

[16] Japanese GastricCancer Association. Japanese classification of gastric carcinoma-3rd English edition. Gastric Cancer 2011; 14: 101-112

[17] Abe S, Oda I, Shimazu T et al. Depth-predicting score for differentiated early gastric cancer. Gastric Cancer 2011; 14: 35-40

[18] Misumi A, Misumi K, Murakami A et al. Endoscopic diagnosis of minute, small and flat early gastric cancers. Endoscopy 1989; 21: 159164

[19] Honmyo U, Misumi A, Murakami A et al. Mechanism producing color change in flat early gastric cancers. Endoscopy 1997; 29: 366 - 371

[20] Sun X, Dong T, Bi Y et al. Linked color imaging application for improving the endoscopic diagnosis accuracy: a pilot study. Sci Rep 2016; 6: 33473

[21] Menon S, Trudgill N. How commonly is upper gastrointestinal cancer missed at endoscopy? A meta-analysis Endosc Int Open 2014; 2: E46-E50

[22] Voutilainen ME, Juhola MT. Evaluation of the diagnostic accuracy of gastroscopy to detect gastric tumors: clinicopathological features and prognosis of patient with gastric cancer missed on endoscopy. Eur J Gastroenterol Hepatol 2005; 17: 1345-1349

[23] Hosokawa O, Hattori M, Douden K et al. Difference in accuracy between gastroscopy and colonoscopy for detection of cancer. Hepatogastroenterology 2007; 54: 442-444

[24] Lee G], Park SJ, Kim SJ et al. Effectiveness of Premedication with Pronase for Visualization of the Mucosa during Endoscopy: A Randomized, Controlled Trial. Clin Endosc 2012; 45: 161 - 164

[25] Machaca QueaNR, Emura F, Barreda BolañosF et al. Effectiveness of systematic alphanumeric coded endoscopy for diagnosis of gastric intraepithelial neoplasia in a low socioeconomic population. Endosc Int Open 2016; 4: E1083-E1089

[26] Kanzaki H, Takenaka R, Kawahara Y et al. Linked color imaging (LCI), a novel image-enhanced endoscopy technology, emphasizes the color of early gastric cancer. Endosc Int Open 2017; 5: E1005-E1013

[27] Yoshifuku Y, Sanomura Y, Oka S et al. Evaluation of the visibility of early gastric cancer using linked color imaging and blue laser imaging. BMC Gastroenterol 2017; 17: 150 